\title{
Un cinéma de poésie, interrogations premières
}

Didier Coureau

\section{(2) OpenEdition}

Journals

Édition électronique

URL : https://journals.openedition.org/recherchestravaux/671

DOI : 10.4000/recherchestravaux.671

ISSN : 1969-6434

\section{Éditeur}

UGA Éditions/Université Grenoble Alpes

\section{Édition imprimée}

Date de publication : 1 novembre 2014

Pagination : 5-11

ISBN : 978-2-84310-282-0

ISSN : 0151-1874

\section{Référence électronique}

Didier Coureau, « Un cinéma de poésie, interrogations premières », Recherches \& Travaux [En ligne], 84 | 2014, mis en ligne le 01 avril 2016, consulté le 29 octobre 2021. URL : http://journals.openedition.org/ recherchestravaux/671; DOI : https://doi.org/10.4000/recherchestravaux.671 
Didier Coureau

Université Grenoble Alpes

\section{Un cinéma de poésie, interrogations premières}

Sur cet écran, qui semblera la projection du feuillet placé sur le rouleau de la machine à écrire, montera en cinématographie le texte du poème, au fur et à mesure de sa déclamation.

Francis PONGE

Un cinéma de poésie, le titre donné au présent volume s'annonce comme une affirmation, celle de l'évidente nécessité de penser une mise en relation entre deux formes de création, l'une usant de l'écriture textuelle, l'autre de l'écriture à l' "encre de lumière" $"$, selon l'expression de Jean Cocteau, "UNE ÉCRITURE AVEC DES IMAGES EN MOUVEMENT ET DES SONS ${ }^{3}$ », d'après Robert Bresson, dont les Notes sur le cinématographe sont autant de fragments poétiques, tout aussi bien que philosophiques, puisqu'ils trouvent leurs propres effets rythmiques, de répétition par exemple. Cocteau ne s’y était pas trompé qui écrivait : "Bresson est "à part” dans ce métier terrible. Il s'exprime cinématographiquement comme un poète par la plume ${ }^{4}$. Mais Cocteau est bien sûr lui-même l'un de ceux qui, le plus rapidement, venant de la poésie textuelle, a pensé que le cinéma était un "véhicule de poésie incomparable" », répondant ainsi au souhait d'Apollinaire de voir s'exprimer des poètes par l'entremise du film, afin de créer un nouveau lyrisme, et suivant la pensée de Jean Epstein qui affirmait pour sa part : «Le

I. F. Ponge, Le Savon, Paris, Gallimard, coll. "L'Imaginaire», 1992, p. 47.

2. J. Cocteau, Du cinématographe, Monaco, Éditions du Rocher, 2003, p. 47.

3. R. Bresson, Notes sur le cinématographe, Paris, Gallimard, I975, p. I2.

4. J. Cocteau, Du cinématographe, ouvr. cité, p. 9I.

5. Ibid., p. 34. 
cinéma est le plus puissant moyen de poésie ${ }^{6}[\ldots]$ ». La trilogie orphique - Le Sang d'un poète, Orphée, Le Testament d'Orphée (années 1930, 1950, 1960) - fait ainsi renaître la figure mythique du poète au sein même du monde contemporain. Dans trois de ses articles, Cocteau tente des alliances entre cinéma et poésie, dès leurs titres : «Le film, véhicule de poésie» (1945 dans Paris-cinéma), «Poésie et films» (1948 dans Film-Kunst), «La poésie au cinématographe» (1949 dans Cinéma). Raoul Ruiz, dans le second tome de son ouvrage Poétique du cinéma retrouve, en un nouveau millénaire, la réflexion de Cocteau et d'Epstein, lorsqu'il écrit : «[...] le cinéma est condamné à être poétique. Il ne peut pas ne pas l'être. On peut ignorer cet aspect de sa nature. Mais la poésie sera toujours là, à portée de sa main. Pourquoi ne pas s'en serviri? ?»

Tout cinéma n'est pas poétique bien sûr, mais la poésie est là, proche, qui peut être révélée par certains cinéastes capables de la percevoir, puis de la faire percevoir. Cette quête de la poésie par Ruiz se retrouve dans cette très belle idée qu'il expose, toujours dans sa poétique : «[...] de film en film nous sommes à la poursuite d'un film secret, lequel se cache parce qu'il ne veut pas être vu. [...] ma thèse est que sans un tel film secret, il n'y a pas d'émotion cinématographique $^{8} »$. La poésie résiderait peut-être au cœur de ce film secret, et serait prise dans un double phénomène d'émergence et de résurgence, pour reprendre des termes chers à Henri Michaux. Il est à remarquer que, quelques années après Ruiz, Eugène Green a lui-même publié un ouvrage intitulé Poétique du cinématographe 9 . Peut-être les années 2000 ont-elles ainsi vu se réactualiser dans les marges la question des relations étroites qui unissent cinéma et poésie. Marges qui entourent les poèmes, "grandes marges blanches [...] grandes marges de silence», dont parlait Paul Éluard dans une conférence au titre évocateur : "L'évidence poétique ${ }^{\mathrm{I} 0}$ », marges "qui font tenir les pages du livre ensemble» comme a pu un jour le déclarer Jean-Luc Godard. Interrogation qui passa par l'avant-garde impressionniste - ainsi dénommée par Henri Langlois - de Louis Delluc, Abel Gance, Jean Epstein, Germaine Dulac, dans les voisinages de Blaise Cendrars; par le surréalisme, côté cinéma dans les films de Man Ray, côté écriture sur le cinéma dans les critiques et les "cinépoèmes" de Robert Desnos ou de Philippe Soupault; puis par la Nouvelle Vague avec les échanges

6. J. Epstein, Écrits sur le cinéma, t. I, Paris, Seghers, coll. «Cinéma club», I974, p. I42.

7. R. Ruiz, Poétique du cinéma, t. I, Miscellanées, Paris, Dis Voir, 2005, p. 2I. Un second tome est paru en 2006 aux mêmes éditions. À l'origine, comme la préface de l'auteur le stipule, il aurait dû y avoir trois volumes sous-titrés Miscellanées, Serio Ludens (Jeux sérieux) et Méthodes.

8. R. Ruiz, Poétique du cinéma, t. I, ouvr. cité, p. Io6.

9. E. Green, Poétique du cinématographe, Arles, Actes Sud, 2009.

IO. P. Éluard, Euvres complètes, Paris, Gallimard, coll. «Bibliothèque de la Pléiade», I979, p. 515 . 
miraculeux qui se produisirent entre Jean-Luc Godard et Louis Aragon ou entre Jean-Daniel Pollet et Francis Ponge.

Le titre du présent volume se présente comme une affirmation, sans être tout à fait identique à l'intitulé de la conférence de Pier Paolo Pasolini, «Le cinéma de poési $\mathrm{e}^{\mathrm{II}} »$. Il convient cependant de revenir sur son texte. Il est possible aujourd'hui d'écarter ce qui concernait trop directement les approches alors privilégiées, ou commençant à s'imposer - sémiologie, narratologie, structuralisme -, pour ne conserver que la réflexion esthétique de Pasolini, qu'il explicite de manière très claire dans un entretien réalisé lors du festival de Pesaro de 1965, où sa conférence "Le cinéma de poésie» fut prononcée.

La ligne directrice du "cinéma de poésie», selon Pasolini, est l'affirmation forte de la présence de la caméra et des effets filmiques qu'elle peut produire :

[...] la langue de la poésie est celle où l'on sent la caméra, de même que dans la poésie proprement dite on sent immédiatement les éléments grammaticaux en fonction poétique; alors que dans la langue de la prose on ne sent pas la caméra, c'est-à-dire qu'en effet on ne sent pas l'effort stylistique comme exprimé, que la présence de l'auteur n'y est pas apparente ${ }^{\mathrm{i} 2}$.

Cette prédominance de la forme éloigne le "cinéma de poésie» des conventions du cinéma narratif :

Il est inévitable que dans le "cinéma de poésie», le récit tende à disparaitre [...]. Il est clair que dans le cinéma de poésie, l'auteur tend à écrire des poésies, des poésies cinématographiques et non plus des récits cinématographiques. Il y a alors valorisation de la poésie, jusqu'ici poésie de la forme et du style. Le "cinéma de poésie» a pour fin dernière d'écrire des récits où le protagoniste est le style, plus que les choses ou les faits ${ }^{13}$.

Si le titre du présent volume n'est pas directement à la forme interrogative - Un cinéma de poésie? -, il devrait néanmoins soulever de multiples questions.

Comment la poésie textuelle s'inscrit-elle dans le film, en influençant dès lors son esthétique, parfois sous la forme d'un véritable surgissement ${ }^{14}$ ? Comment la rencontre avec un poète et sa poésie peut-elle conférer une dimension poétique à un film? Comment l'image filmique entre-t-elle en coïncidence avec

II. P. P. Pasolini, «Le cinéma de poésie», dans L'Expérience hérétique, Paris, Ramsay, coll. "Poche cinéma", 1989, p. 15-35.

I2. P. P. Pasolini dans «Entretien avec Pasolini», par J.-L. Comolli et B. Bertolucci, Pasolini cinéaste, numéro hors-série des Cahiers du cinéma, I98I, p. 35 ( $\mathrm{I}^{\mathrm{re}}$ publication dans les Cahiers $d u$ cinéma, $\mathrm{n}^{\circ} \mathrm{I} 69$, août 1965$)$. La conférence, "Le cinéma de poésie " peut-être lue dans L'Expérience hérétique, Paris, Ramsay, coll. "Poche cinéma», 1989, p. 15-35.

13. Ibid., p. 39.

I4. Voir D. Coureau, "Avez-vous entendu parler des messages secrets?», dans Cinéma et Littérature, le grand jeu, J.-L. Leutrat (dir.), Grenoble, De L'Incidence, 2010. 
l'image poétique, dans un rapport au mot et à sa diction mais aussi, au-delà, en rejoignant un certain esprit de poésie - un livre essentiel de Jean Epstein s'intitule Esprit de cinéma -, état de conscience dans la perception du réel, ou de subconscient dans l'exploration du surréel? Comment le regard porté sur les êtres et le monde se trouve-t-il métamorphosé poétiquement par les puissances métaphoriques du cinéma? Comment l'expérience intérieure du cinéaste rejoint-elle l'expérience intérieure du poète? Pourquoi le poète veutil s'exprimer par les moyens du film? Comment la forme filmique peut-elle devenir poétique, et échapper aux règles conventionnelles de la narration, par la création d'un rythme propre, ainsi que le pressentait déjà Germaine Dulac lorsqu'elle écrivait :

Le cinéma peut certes raconter une histoire, mais il ne faut pas oublier que l'histoire n'est rien. L'histoire, c'est une surface. Le septième art, l'art de l'écran, c'est la profondeur rendue sensible qui s'étend au-dessus de cette surface : l'insaisissable musical ${ }^{15}$.

ou encore :

La définition de l'action cinématographique, qui nous la donnera? Sensations peut-être plus que suite de faits... Sensations de musique, de philosophie et de poésie, plus que de drames ${ }^{16}$.

Par «un cinéma de poésie», il est aussi permis d'entendre que la réflexion puisse porter sur la manière dont le texte poétique s'imprègne de correspondances avec l'art cinématographique (images et sons, montage), de se demander comment le poème peut se faire film virtuel à sa manière dans la fusion et la confusion des images nées des mots et les dépassant, en une approche renouvelée de l'espace et du temps.

Les quatre sections de l'ouvrage reprennent en quelque sorte, en les synthétisant, ces interrogations : "Le cinéma et les poètes», "Cinéma poétique, poétique du cinéma», "Une poésie cinématographique», "Poète spectateur, Poète filmeur, Cinéaste poète».

Louverture à différents horizons géographiques fait également partie des lignes directrices qui se sont progressivement dessinées, et elle peut influer sur ces différents questionnements concernant les relations entre cinéma et poésie, poésie et cinéma. Ici, le voyage se fait dans l'Angleterre romantique de John Keats vue par la réalisatrice néo-zélandaise Jane Campion, Brightstar (Esra Aykin), le Portugal de Fernando Pessoa et de Manoel de Oliveira (Guillaume

I5. G. Dulac, Écrits sur le cinéma (I9I9-1937), P. Hilairet (éd.), Paris, Paris expérimental, I994, p. 108 .

16. Ibid., p. 52. 
Bourgois), le Japon de Yasujiro Ozu (Thomas de Davydoff), les États-Unis de Maya Deren (Olivier Salazar-Ferrer, par ailleurs spécialiste, entre autres poètes, de Benjamin Fondane, qui fut aussi théoricien du cinéma et cinéaste $\left.{ }^{17}\right)$, l'Afrique de François L. Woukoache, la déterritorialisation d'un cinéaste d'origine américaine, Eugène Green (Didier Coureau), qui se fit naturaliser français par amour de la langue.

Les contributions réunies ne se cantonnent pas plus à un lieu qu'à un temps donné, et des cinéastes de plusieurs périodes sont abordés. De grands réalisateurs de l'histoire du cinéma mondial, comme Yasujiro Ozu aujourd'hui disparu, ou Manoel de Oliveira encore en vie bien qu'ayant traversé tout le siècle précédent, sont ainsi évoqués. Le cinéma expérimental américain est représenté par Maya Deren (qui commença à filmer dans les années I940), dont l'esthétique est marquée par celle de Jean Cocteau. Du côté du cinéma contemporain sont étudiés des réalisateurs de registres différents; le film de Jane Campion qui porte un regard biographique sur un grand poète romantique anglais, Eugène Green qui constitue sa propre poésie filmique, imprégnée par les siècles littéraires et artistiques antérieurs au XviII ${ }^{\mathrm{e}}$ siècle et les grands cinéastes français du $\mathrm{xx}^{\mathrm{e}}$ siècle, Jean-Noël Cristiani (Le Marcheur) dans un cheminement spirituel très personnel. Pour ce qui concerne la relation des poètes au cinéma, sont approchés Stéphane Mallarmé et Francis Ponge autour d'une «kinoptique» qui préfigure la projection cinématographique, sur un ciel-écran, dans l'interprétation de Christophe Wall-Romana - auteur par ailleurs d'un ouvrage monumental sur la relation des poètes français au cinéma (Cinepoetry, Imaginary Cinemas in French Poetry, New York, Fordham University Press, 20I3) - et, du côté de l'extrême contemporain, par Catherine Soulier, une poésie textuelle née du cinéma venue "contaminer» l'écriture d'Ariane Dreyfus, Jérôme Game et Pierre Alféri.

Si les contributions prennent, pour la plupart, une forme universitaire, il n'a pas été exclu d'intégrer des formes d'écritures qui entremêlaient la pensée et la création, comme autant de lignes de fuite toujours salutaires.

Jacques Laurans, écrivain, poète, a écrit épisodiquement, entre la critique et la prose poétique, sur le cinéma et sur des films, livrant des textes sur Robert Bresson, Michelangelo Antonioni, Wim Wenders. Il a récemment publié un livre intitulé $O z u$ père éternel (Hermann, 2013) que cite en certains de ses passages le texte de Thomas de Davydoff, également consacré à Ozu. Le récit autobiographique de Laurans, Dans la salle obscure (Seuil, 1997), évoquait sa découverte du cinéma, lors de sa jeunesse à Casablanca. C’est peut-être autour

17. Voir B. Fondane, Écrits pour le cinéma (le muet et le parlant), textes réunis et présentés par M. Carassou, O. Salazar-Ferrer, R. Fotiade, Lagrasse, Verdier, coll. «Poche», 2007. 
d'un intérêt commun pour Pierre Soulage que Laurans a rencontré le cinéaste Jean-Noël Cristiani qui a consacré deux films au plasticien : Les Vitraux de Soulages et Soulages, le noir et la lumière. Cristiani, s'est aussi intéressé par ailleurs à des écrivains dans ses films-essais (Mika Waltari, Karen Blixen, Édouard Glissant, Maurice Chappaz). Mais le film ici traité concerne l'expérience plus intime, et non dénuée de spiritualité, du marcheur.

Gérard Leblanc qui fut professeur en études cinématographiques à l'université Paris 3, puis à l'École nationale supérieure Louis Lumière, est aussi poète - activité qu'il pratique sous le nom de Gérard Soler. Dans son ouvrage Trajectoires (L'CEil, 200I), il entremêle poésies, textes sur la poésie écrite, et textes sur la poétique du cinéma. Il faut aussi signaler, aux mêmes éditions, un très bel ouvrage, L'EntreVues (1998), qu'il a composé avec le cinéaste JeanDaniel Pollet. Leblanc réfléchit dans le présent volume sur sa propre pratique vidéo-filmique, dans la collaboration avec sa compagne Catherine Guéneau, à travers une poétique de la relation amoureuse (de la rencontre à la naissance d'un enfant).

Frank Smith ne conçoit pas la poésie comme détachée du monde, de ses bouleversements historiques perpétuels, comme le démontre l'un de ses derniers ouvrages, Gaza, d'ici là (Al Dante, 2013). S’il est venu au cinéma, cela semble être dans une prolongation naturelle de son œuvre poétique. Eureka (2009), dont le titre évoque une ville des États-Unis, peut aussi faire songer au célèbre poème d'Edgar Poe, «Eurêka» - dont Jean-Louis Schefer a d'ailleurs étudié les points de jonction avec le cinéma dans un très beau texte ${ }^{\mathrm{r}}$. Le film dont il est ici question fait entendre les voix off d'Aurore Clément, Tchéky Kario, et Michaël Lonsdale, autant de comédiens liés à un cinéma différent : Chantal Akerman, Eric Rohmer, Marguerite Duras. Son expérience filmique la plus récente porte un titre qui évoque Edmond Jabès : Le Film des questions.

Si à l'inverse de Frank Smith, François L. Woukoache est avant tout cinéaste, son cinéma tend, dans son film Asientos (réalisé en 1995, alors qu'il n'avait que vingt-neuf ans), vers une poésie - alliance du texte, du son, de la plastique visuelle - où se mêlent également regard sur le monde et réflexion sur la mémoire. Est ici donné principalement à lire le texte des voix off d'Asientos, où la mémoire de la traite des Noirs communique avec la source essentielle que constitue le recueil manifeste d'Aimé Césaire, Cahier d'un retour au pays natal, ainsi qu'avec l'esthétique et les problématiques des films d'Alain Resnais

I8. J.-L. Schefer, Du Monde et du mouvement des images, Paris, Cahiers du cinéma, coll. "Essais», I997. Aux côtés de Poe, sont analysés dans cet ouvrage Giacomo Leopardi et Jean Epstein. Il s’agit bien de montrer comment la poésie a pu annoncer ce que le cinéma serait capable de donner à voir, le temps dans l'image, la matière en mouvement, une forme de devenir-cosmique - de l'image - ainsi qu'auraient pu le formuler G. Deleuze et F. Guattari. 
(Nuit et Brouillard et Hiroshima mon amour), Chris Marker, Marguerite Duras, Jean-Daniel Pollet et, du côté africain, Souleyman Cyssé.

Un cinéma de poésie prolonge un travail entrepris au sein de l'équipe de recherche Traverses 19-2I de l'université Stendhal Grenoble Alpes, et de son pôle CinestheA, sur les relations entre cinéma et poésie, lors de journées d'études en novembre 20I0, intitulées "Cinéma, Arts vidéo, Poésie : les images au miroir des images» (sous la direction de Didier Coureau) qui ont par la suite donné lieu à un numéro hors-série de la revue Murmures (Grenoble, De L'Incidence, à paraître), sous le titre Poésie en projection - certains textes étant venus s'adjoindre dans l'intervalle aux contributions originales. Cette réflexion n'est pas vouée à s'interrompre avec ces deux publications qui veulent, bien au contraire, constituer des ouvertures sur l'avenir ${ }^{19}$.

Un dernier mot s'impose sur la forme même de l'ouvrage. L'absence de toute illustration, de tout photogramme filmique, donne non seulement une unité à l'ensemble, mais signifie aussi qu'il est ici fait confiance aux mots et à leur pouvoir de donner à penser, mais aussi de donner à voir et de donner à entendre - lorsque les mots disent les images et les sons. Un photogramme ne procure-t-il pas une vision faussée d'un film, en interrompant son flux au sein de l'Image-Temps?

I9. Il faut encore mentionner un récent colloque qui eut lieu à l'université Montpellier 3, en mars 20I3, intitulé "Cinéma et Poésie», organisé par C. Soulier et M. Poirson-Dechonne pour l'équipe de recherche RIRRA 2I, et qui doit également donner lieu à la publication prochaine d'un ouvrage. 